\title{
YL4073 is a potent autophagy-stimulating antitumor agent in an in vivo model of Lewis lung carcinoma
}

\author{
YOU-ZHI XU ${ }^{1 *}$, YONG-HUAI LI ${ }^{2 *}$, WEN-JIE LU ${ }^{1 *}, \mathrm{KUN} \mathrm{LU}^{4}$, CHUN-TING WANG ${ }^{3}$, YAN LI $^{3}$, \\ HONG-JUN LIN ${ }^{3}$, LI-XIN KAN ${ }^{1}$, SHENG-YONG YANG ${ }^{3}$, SI-YING WANG ${ }^{1}$ and YING-LAN ZHAO ${ }^{3}$ \\ ${ }^{1}$ School of Basic Medicine, Anhui Medical University, Hefei, Anhui 230032; ${ }^{2}$ Department of Respiratory Disease, \\ The First Affiliated Hospital of Anhui Medical University, Hefei, Anhui 230022; ${ }^{3}$ State Key Laboratory of Biotherapy \\ and Cancer Center, West China Hospital, Sichuan University, Collaborative Innovation Center for Biotherapy, \\ Chengdu, Sichuan 610041; ${ }^{4}$ Department of Electrocardiogram, The First Affiliated Hospital \\ of Anhui Medical University, Hefei, Anhui 230022, P.R. China
}

Received October 21, 2015; Accepted December 7, 2015

DOI: 10.3892/or.2016.4603

\begin{abstract}
Cancer cells activate autophagy in response to anticancer therapies. Autophagy induction is a promising therapeutic approach to treat cancer. In a previous study, YL4073 inhibited the growth of liver cancer and induced liver cancer cell apoptosis. Here, we demonstrated the anticancer activity and specific mechanisms of YL4073 in Lewis lung carcinoma LL/2 cells. Our results show that YL4073-induced autophagy was followed by apoptotic cell death. The anticancer and autophagy stimulating efficacy was confirmed by several factors, including the appearance of autophagic vacuoles, formation of acidic vesicular organelles, recruitment of microtubule-associated protein 1 light chain 3 II (LC3-II) to the autophagosomes, conversion and cleavage of LC3-I to LC3-II, upregulation of Beclin 1 expression, and formation of the Atg12-Atg5 conjugate in LL/2 cells after YL4073 treatment for 24 or $48 \mathrm{~h}$. Furthermore, P53 activation and p-histone $\mathrm{H} 3$ phosphorylation occurred after cell exposure to YL4073 for $48 \mathrm{~h}$, suggesting that cell apoptosis had occurred. Pharmacological inhibition of autophagy using 3-methyladenine increased cell apoptosis. Molecular level studies revealed
\end{abstract}

Correspondence to: Professor Ying-Lan Zhao, State Key Laboratory of Biotherapy and Cancer Center, West China Hospital, Sichuan University, Collaborative Innovation Center for Biotherapy, 17 Ren Min South Road, 3rd Section, Chengdu, Sichuan 610041, P.R. China

E-mail: zhaoyinglan@scu.edu.cn

Professor Si-Ying Wang, School of Basic Medicine, Anhui Medical University, 81 Mei Shan Road, Hefei, Anhui 230032, P.R. China

E-mail: sywang@ahmu.edu.cn

*Contributed equally

Key words: YL4073, anticancer therapies, Lewis lung cancer LL/2 cell, microtubule-associated protein 1 light chain 3, autophagy, apoptosis that YL4073 inhibited survival signalling by blocking the activation of Akt and mTOR phosphorylation and reduced the expression of $\mathrm{p}$-mTOR downstream targets for phosphorylation, including p70S6K, p-TSC, p-MAPK, and p-AMPK. This suggests that the Akt/mTOR/p70S6K and TSC/MAPK/AMPK pathways are involved in the effects of YL4073 treatment in LL/2 cells. In addition, YL4073 significantly inhibited LL/2 tumor growth and induced apoptosis in vivo. These data suggest that YL4073 has a significant anticancer effect, with a pathway-specific mechanism of autophagy both in vitro and in vivo.

\section{Introduction}

Lung cancer, the most common type of cancer, represents a major public health problem worldwide $(1,2)$. Lung cancers have been ranked as the main cause of cancer-related morbidity and mortality (3-5). Unfortunately, lung cancer therapy has not been explored extensively in the field of pharmaceutics (6). Because of the poor outcomes associated with lung cancer, drugs with high efficacy are needed to treat this malignancy (7-9).

Small molecular agents have previously exhibited efficacy in anticancer therapies $(10,11)$. YL4073 has thus far exhibited a profound effect on liver cancer (12). It is possible that YL4073 may have novel pharmacological application as a medicinal product with inhibitory activity in other cancers, such as Lewis lung cancer.

Autophagy could induce cell death and is activated in response to stress and nutrient deprivation (13). Autophagy is a complex catabolic mechanism of lysosomal degradation of proteins and other sub-cellular constituents (14-16). Several signaling pathways have been identified involved in cancer associated with the response to autophagy $(17,18)$. Although autophagy has different roles in the modulation of cancers $(19,20)$, it is known as a mechanism of cell death and tumor suppression (21-23).

In the present study, we identified YL4073 as a potent anticancer agent capable of inducing tumor cell autophagy. We focused our research mainly on lung carcinoma, owing to its 
poor prognosis and lack of effective therapies for this tumor type in clinical settings, in an attempt to provide preclinical study profiles for clinical treatment. We have shown that YL4073, a small molecular agent, was a potent targeted autophagy associated protein, which induced autophagy in LL/2 cells. Cell autophagy was followed by apoptosis indicating the involvement of $\mathrm{Akt} / \mathrm{m}-\mathrm{TOR} / \mathrm{p} 70 \mathrm{~S} 6 \mathrm{~K}$ and TSC/MAPK/AMPK pathways. In addition, YL4073 significantly inhibited the growth of LL/2 tumors in vivo. Our results indicated that YL4073 has significant anticancer activity and autophagy was suggested to be involved in this activity. To the best of our knowledge, this study was the first to demonstrate that YL4073 was able to induce autophagy in cancer cells.

\section{Materials and methods}

Materials. Dimethyl sulfoxide (DMSO), propidium iodide (PI), acridine orange, Z-VAD-FMK, and 3-methyladenine (3-MA) were purchased from Sigma Chemical Co. (St. Louis, MO, USA). Cell Counting Kit-8 (CCK-8) was purchased from Dojindo Laboratory (Dojin, Japan); all chemicals employed in this study were of pure analytic and culture grade. The primary antibodies for LC-3/LC3-II, Beclin 1, Atg5/Atg12, p-mTOR, p-Akt, p-P70S6K, p-TSC, p-AMPK, p-p44/42 MAPK, P53, PTEN, and p-Histone H3 were purchased from Cell Signaling Technology (Beverly, MA, USA); horseradish peroxidase (HRP)-conjugated anti-rabbit/mouse secondary antibodies were purchased from Santa Cruz Biotechnology (Santa Cruz, CA, USA). $\beta$-actin and GAPDH were obtained from Boster Biotechnology (Wuhan, China). Protein assay kit was purchased from Bio-Rad (Hercules, CA, USA). For all in vitro assays, YL4073 and 3-MA were dissolved in DMSO to prepare a stock solution of $40 \mathrm{mM}$ and $5 \mathrm{M}$, respectively, and stored at $4^{\circ} \mathrm{C}$. The stock solutions were diluted in the relevant media to the final DMSO concentration of $0.05 \% \mathrm{v} / \mathrm{v}$ when used. For all in vivo studies, YL4073 was suspended in ultrapure water and Cremophor EL/ethanol (50:50, Cremophor EL, 95\% ethyl alcohol; Sigma Chemical Co.) and administered at $10 \mathrm{ml} / \mathrm{kg} /$ day of body weight by intraperitoneal injection.

Cell culture. Murine Lewis lung carcinoma LL/2, mammary carcinoma cell 4T1, fibroblast NIH-3T3, and human proximal tubular cell human kidney-2 (HK-2) cells were obtained from the American Type Culture Collection (ATCC; Manassas, VA, USA) and cultured in RPMI-1640 or Dulbecco's modified Eagle's medium (DMEM; Life Technologies, Bedford, MA, USA) containing $10 \%$ heat-inactivated FBS (Gibco-BRL, Grand Island, NY, USA), $100 \mathrm{U} / \mathrm{ml}$ penicillin, and streptomycin in a humid chamber at $37^{\circ} \mathrm{C}$ and $5 \% \mathrm{CO}_{2}$.

Cell viability by $C C K-8$ assay. Cells were plated in 96-well plates for $24 \mathrm{~h}$ and cultured with YL4073 for $72 \mathrm{~h}$; $10 \mu \mathrm{l} \mathrm{CCK}-8$ solution was added to each well. The $\mathrm{IC}_{50}$ of YL4073 was calculated and optical density (OD) was measured at $450 \mathrm{~nm}$ with a Multiskan Spectrum instrument (Thermo Lab Systems, USA) after cells were incubated with CCK-8 for $2-4 \mathrm{~h}$ at $37^{\circ} \mathrm{C}$.

EdU-DNA incorporation assay. 5-Ethynyl-2'-deoxyuridine $(E d U)$ is a nucleoside analogue of thymidine, which can be incorporated into DNA during DNA synthesis. An EdU-DNA incorporation assay kit (RiboBio, China) was used to detect the percentage of uptake in proliferating LL/2 cells. Briefly, $3 \times 10^{3} \mathrm{LL} / 2$ cells were seeded in 96-well plates for $24 \mathrm{~h}$ and treated with YL4073 for $48 \mathrm{~h}$. Then, the cells were incubated with EdU for $2-3 \mathrm{~h}$ at $37^{\circ} \mathrm{C}$, and the plates observed with an inverted fluorescent microscope (Carl Zeiss, Germany). EdU-positive cells were counted in five fields per well; the average was calculated according to the following formula: EdU $(\%)=($ EdU-positive cells $) /($ Hoechst-positive cells $) \times 100$. The experiment was done in triplicate.

Detection of acidic vesicular organelles. For detection of acidic vesicular organelles (AVO) in vitro (24), $1 \times 10^{5} \mathrm{LL} / 2$ cells were plated in 6-well plates and treated with $20 \mu \mathrm{M}$ YL4073 or $2 \mathrm{mM} 3$-MA for $48 \mathrm{~h}$. The cells were then stained with $1 \mu \mathrm{g} / \mathrm{ml}$ acridine orange for $15 \mathrm{~min}$, after which images were obtained under a fluorescent microscope equipped with a digital camera $(25,26)$.

Autophagy and apoptosis analysis by flow cytometry (FCM). To further confirm autophagy of the cells, we first analyzed LL/2 cells by FCM after AVO staining. Cell culture and drug treatment were carried out as described above. After being treated with $1 \mu \mathrm{g} / \mathrm{ml}$ AVO for $10 \mathrm{~min}$, cells were collected and immediately analyzed by FCM (Beckman Coulter, Miami, FL, USA). Furthermore, LL/2 cells were treated with YL4073 for $48 \mathrm{~h}$. Cells were collected and incubated with $1 \mathrm{ml}$ hypotonic fluorochrome solution containing $50 \mathrm{ng} / \mathrm{ml} \mathrm{PI}$ in $0.1 \%$ sodium citrate plus $0.1 \%$ Triton X-100, and were immediately analyzed by FCM. Finally, the cell-permeable caspase inhibitor Z-VAD-FMK and autophagy inhibitor 3-MA were used to study whether caspase family protein kinases and/or autophagy was involved in YL4073-induced apoptosis and autophagy. For this assay, LL/2 cells were treated with $20 \mu \mathrm{M}$ YL4073 combined with $10 \mathrm{mM}$ Z-VAD-FMK or $2 \mathrm{mM} 3-\mathrm{MA}$, respectively. PI staining was performed and analyzed by FCM 24 and $48 \mathrm{~h}$ later.

Western blot analysis. Standard western blot analysis was performed to identify the possible mechanism of YL4073. Briefly, LL/2 cells were treated by YL4073 for $48 \mathrm{~h}$ and cell proteins were extracted. Western blotting was further examined via electrophoretic transfer of sodium dodecyl sulphate-polyacrylamide gel electrophoresis (SDS-PAGE), separation of proteins on polyvinylidene fluoride (PVDF) membranes (Millipore, Billerica, MA, USA), and incubation with primary and secondary antibodies. Protein bands were visualized using an enhanced chemiluminescence kit (Amersham Biosciences Corp., Piscataway, NJ, USA).

Pharmacokinetics analysis of YL4073 in SD rats. SD male rats (weight, 180-200 g) were obtained from the Beijing Animal Center (Beijing, China) received an intraperitoneal injection with a single dose of $30 \mathrm{mg} / \mathrm{kg}$ YL4073 and the pharmacokinetic profiles were determined by high-pressure liquid chromatography (HPLC; Waters, USA). Briefly, the blood of $\mathrm{SD}$ rats $(n=3)$ was collected in heparinized tubes at designated time-points after drug administration and centrifuged immediately to separate plasma from blood cells. Samples $(90 \mu \mathrm{l})$ were mixed with $100 \mu \mathrm{l}$ of acetonitrile containing $10 \mu \mathrm{l}$ internal standards $(5 \mu \mathrm{g} / \mathrm{ml})$ and centrifuged at $6,000 \mathrm{x} \mathrm{g}$ for $10 \mathrm{~min}$. 

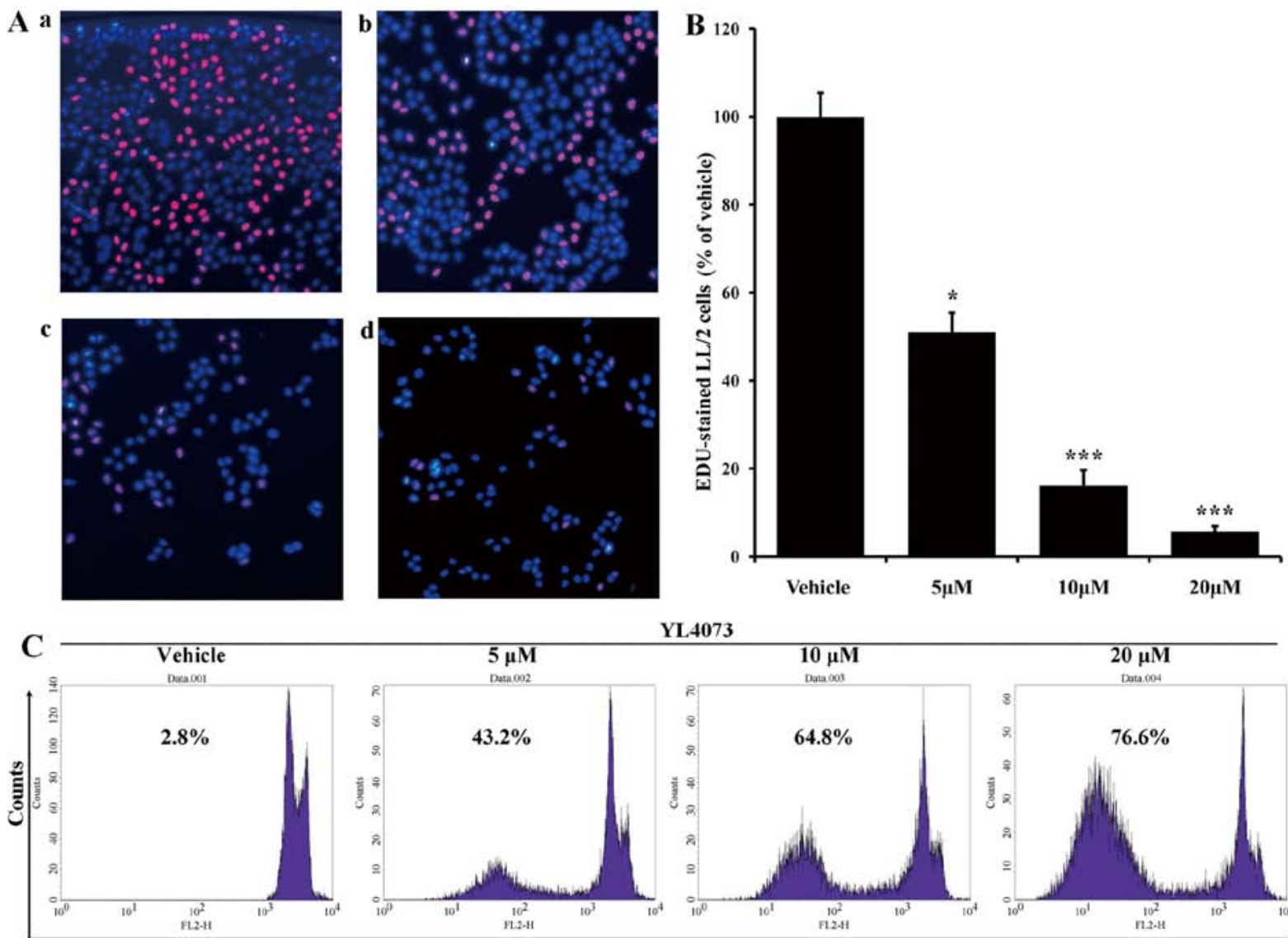

YL4073
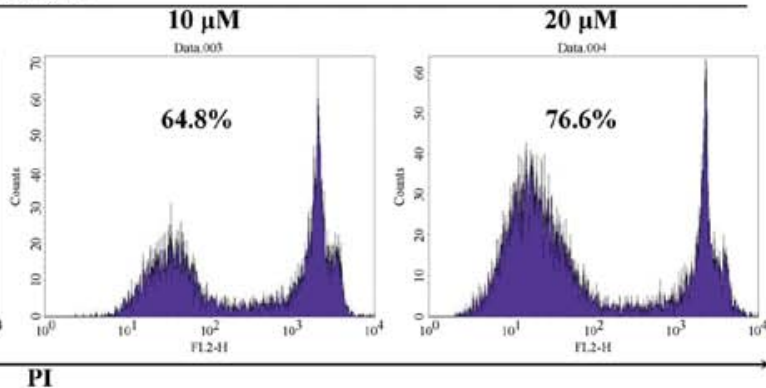

Figure 1. YL4073 inhibited cellular proliferation and induced apoptosis in vitro. (A) The EdU-DNA incorporation assay using YL4073-treated LL/2 cells. Staining with Hoechst 333258 and EdU in LL/2 cells treated with YL4073 for 48 h (x100 magnification). (B) The EdU-positive cells were analysed using Image analyzing software. Data were expressed as mean $\pm \mathrm{SD} ; \mathrm{n}=3 ;{ }^{*} \mathrm{P}<0.05 ;{ }^{* * *} \mathrm{P}<0.001$. (C) YL4073 induced LL/2 cell apoptosis (concentration-dependent manner). Cells were incubated with YL4073 for 48 h, PI-stained, and analyzed by FCM. Data are representative of three parallel experiments.

Table I. The effects of YL4073 on tumor cell viability.

\begin{tabular}{llc}
\hline Cell line & \multicolumn{1}{c}{ Cell type } & $\begin{array}{c}\mathrm{IC}_{50} \\
(\mu \mathrm{M})\end{array}$ \\
\hline LL/2 & Murine Lewis lung carcinoma cell line & 5.29 \\
4T1 & Mouse mammary carcinoma cell line & 9.33 \\
NIH-3T3 & Mouse fibroblasts cell line & $>40$ \\
HK-2 & Human proximal tubular cell line & $>40$ \\
\hline
\end{tabular}

CCK-8 assay was employed to detect the cell viability. Each cell line was treated with various concentrations of YL4073 for $72 \mathrm{~h}$, respectively. Data are expressed as the mean from three experiments.

Then, $100 \mu \mathrm{l}$ supernatant was evaporated and re-dissolved in a solution of $50 \mu \mathrm{l}$ acetonitrile: water $50: 50 \mathrm{v} / \mathrm{v}$ containing $10 \%$ formic acid and subjected to HPLC. Pharmacokinetic parameters were analyzed using a Pharmacokinetic software of Drug and Statistics (DAS, edited by Mathematical Pharmacology Professional Committee of China, version 2.1.1). All animal experiment protocols were conducted in full compliance with our universities for the Care and Use of Laboratory Animals and Experimental Animal Ethics Committee.
Pharmacodynamic analysis of YL4073 in vivo. Female C57BL/6 mice (6-8 week-old) were obtained from the Beijing Animal Center (Beijing, China) and used in the present study. Each mouse received a single injection of harvested LL/2 cells on the flank in the axillary region for the subcutaneous mouse tumor model (27). The tumors were allowed to grow for ten days, and the animals were subsequently sorted into groups of ten for treatment. Tumor volume was measured in two dimensions with vernier calipers and calculated using the following formula: Tumor volume $=\left(\right.$ length $\mathrm{x}$ width $\left.{ }^{2}\right) \times 0.5(28)$.

TUNEL assay in vivo. TUNEL [terminal deoxyribonucleotidyl transferase (TDT)-mediated dUTP-digoxigenin nick end labeling] assay was performed to examine the apoptosis induction effect of YL4073 on LL/2 cells in vivo. LL/2 tumor sections from vehicle and YL4073-treated mice were subjected to TUNEL assay according to the manufacturer's instruction. TUNEL-positive cells were counted under a microscope in three equal-sized fields per slide for quantitative analysis of apoptotic cells. The percentage of apoptotic cells was evaluated as follows: Tumor apoptotic index $(\%)=$ apoptotic cells/ total cells x 100 (12).

Immunohistochemical analysis in vivo. Immunohistochemical analysis was used to detect the autophagy induction effect of 


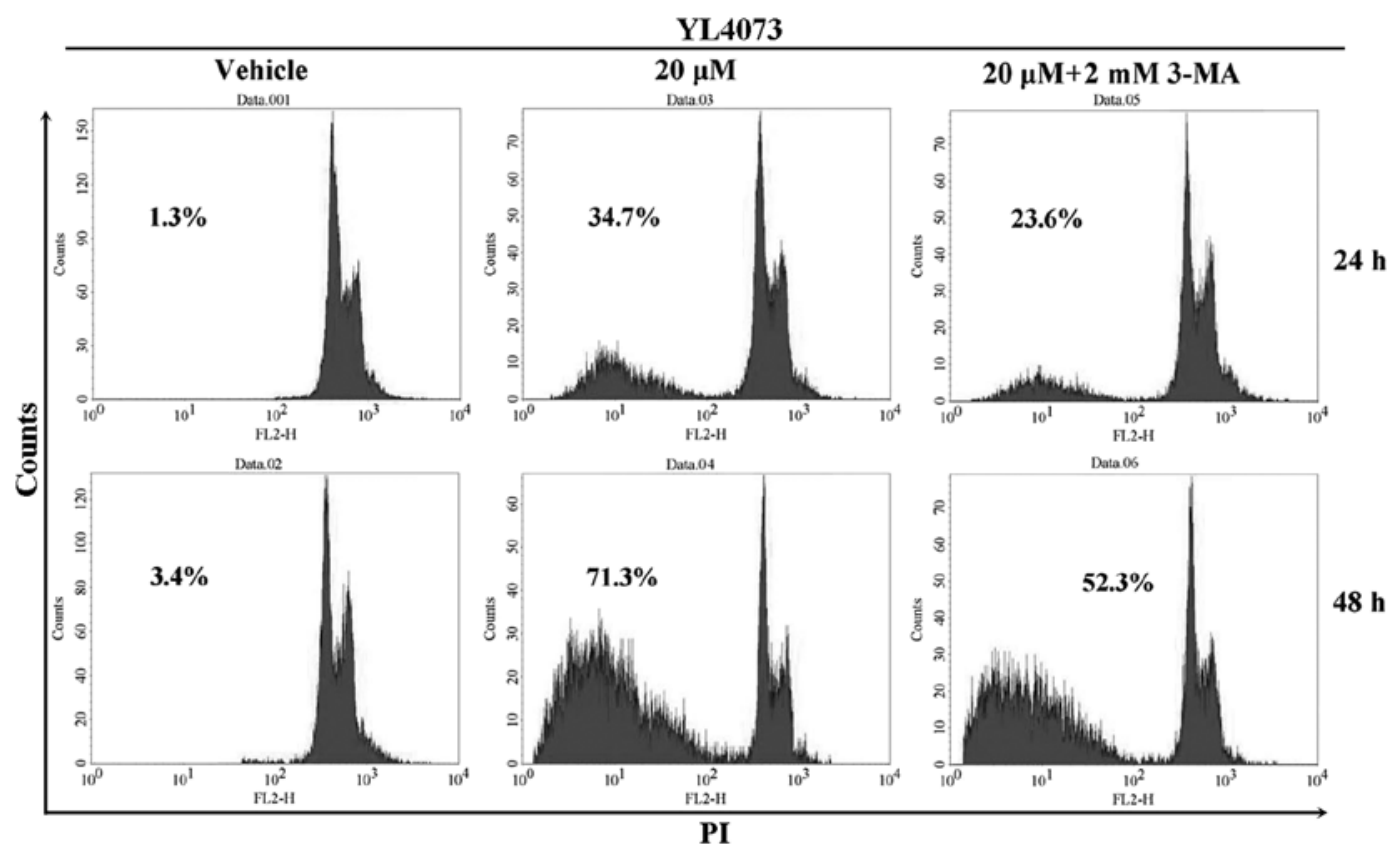

Figure 2. The autophagy inhibitor 3-MA antagonized YL4073-induced apoptosis in vitro. LL/2 cells were incubated with YL4073 and 3-MA and analysed by FCM at 24 and $48 \mathrm{~h}$, respectively.

YL4073 on LL/2 cells in vivo. LL/2 tumor sections from vehicle and YL4073-treated mice were incubated with primary LC3-II antibody and the corresponding second antibody according to the manufacturer's instruction. Representative images were then observed under an inverted microscope with a camera system, in three equal-sized fields per slide (Carl Zeiss) (12).

Statistical analysis. The data were analyzed by SPSS 13.0 software (SPSS, Inc., Chicago, IL, USA) and presented as mean \pm SD/SEM. Kaplan-Meier curves were used to analyze survival and tested with a log-rank test.

\section{Results}

YL4073 inhibited proliferation of murine cancer cells in vitro. CCK-8 assay was used to measure the effect of YL4073 on cell viability. Our results showed that YL4073 decreased the viability of $4 \mathrm{~T} 1$ and $\mathrm{LL} / 2$ cell lines with $\mathrm{IC}_{50}$ values of 9.33 and $5.29 \mu \mathrm{M}$ after treatment for $72 \mathrm{~h}$, respectively (Table I). LL/2 cells were used to further study the mechanism of YL4073 in Lewis lung carcinoma. The $\mathrm{IC}_{50}$ for NIH-3T3 and HK-2 cell lines was $>40 \mu \mathrm{M}$, higher than that for the cancer cell line.

Anti-proliferation effects of YL4073 in LL/2 cells in vitro. The EdU-DNA incorporation assay validated the anti-proliferation ability of YL4073. As shown in Fig. 1B and C, the percentage of EdU-positive cells was $51.05 \%$ after $5 \mu \mathrm{M}$ YL4073 compared with that after vehicle treatment, whereas the percentage of EdU-positive cells decreased to $16.18 \%$ and $5.67 \%$ when cells were treated with 10 and $20 \mu \mathrm{M}$ YL4073, respectively.

Effects of YL4073 on LL/2 cell apoptosis in vitro. Morphological changes of LL/2 cells (stained with PI) were assessed after treatment with YL4073. As shown in Fig. 1, the percentage of sub-G1 cells in the YL4073-treated group increased in a concentration-dependent manner. After cells were treated with $5 \mu \mathrm{M} \mathrm{YL4073,} \mathrm{the} \mathrm{apoptosis} \mathrm{rate} \mathrm{was} 43.20 \%$; the rate of apoptosis increased to $64.80 \%$ and $76.60 \%$ after cells were treated with 10 and $20 \mu \mathrm{M}$ YL4073 for $48 \mathrm{~h}$, respectively.

Effect of caspase on YL4073-induced apoptosis. The effect of autophagy inhibitor 3-MA on YL4073-induced apoptosis was assayed by FCM. The rate of apoptosis after treatment with $20 \mu \mathrm{M}$ YL4073 plus $2 \mathrm{mM} 3-\mathrm{MA}$ decreased from 34.7 to $23.6 \%$ and from $71.3 \%$ to $52.3 \%$ after 24 and $48 \mathrm{~h}$ treatment, respectively, compared with that after YL4073 treatment alone (Fig. 2). Furthermore, similar results were observed in caspase inhibitor Z-VAD-FMK-treated LL/2 cells (data not shown). These results suggest that YL4073-induced apoptosis in LL/2 cells is associated with autophagy, which may be one of mechanisms of the anticancer effect of YL4073.

Induction of autophagy in LL/2 cells examined with AVO assay. Cell death by autophagy has been proposed recently $(29,30)$. Autophagy is characterized by AVO formation, which was detected and measured by staining with acridine orange $(31,32)$. As shown in Fig. 3A, staining LL/2 cells with acridine orange showed the accumulation of AVO in the cell cytoplasm after exposure to $20 \mu \mathrm{M}$ YL4073. This was inhibited by addition of $2 \mathrm{mM} 3$-MA, which inhibited autophagosome sequestration $(33,34)$. FCM analysis was used to quantify the YL4073-induced increase in the fractional volume and acidity of AVO. As shown in Fig. 3B, $20 \mu \mathrm{M}$ YL4073 increased the intensity of fluorescence in LL/2 cells to $85.99 \%$ compared with vehicle, indicating that $2 \mathrm{mM} 3$-MA suppressed the development of AVO in LL/2 cells. YL4073 significantly affected development of AVO in $\mathrm{LL} / 2$ cells compared with vehicle $(\mathrm{P}<0.01)$, and the inhibitory effect of 3-MA on development of AVO was also significant $(\mathrm{P}<0.05)$. 

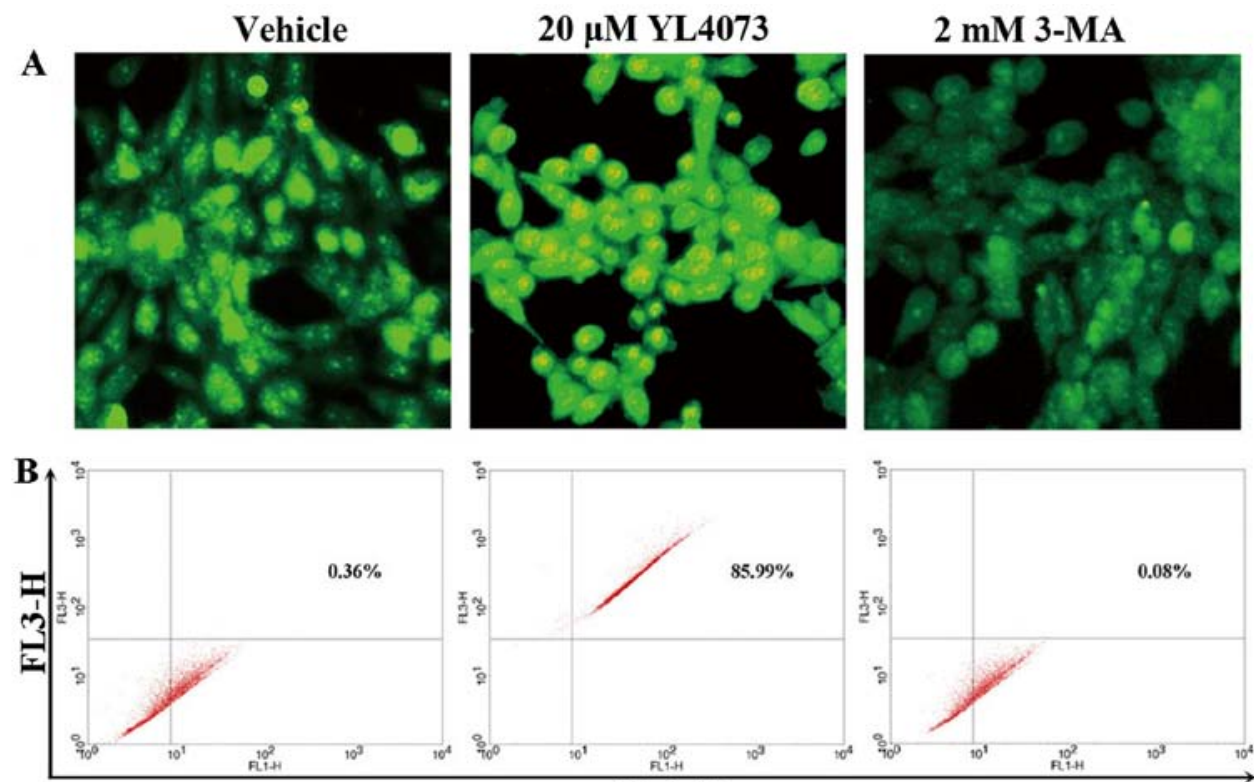

FL1-H

Figure 3. Detection and quantification of AVO with acridine orange staining. (A) Fluorescence microscopic detection of AVO in LL/2 cells after treatment for $48 \mathrm{~h}$ and acridine orange staining (x100 magnification). (B) Quantification of cells with enhanced fluorescence intensity using FCM after acridine orange staining.

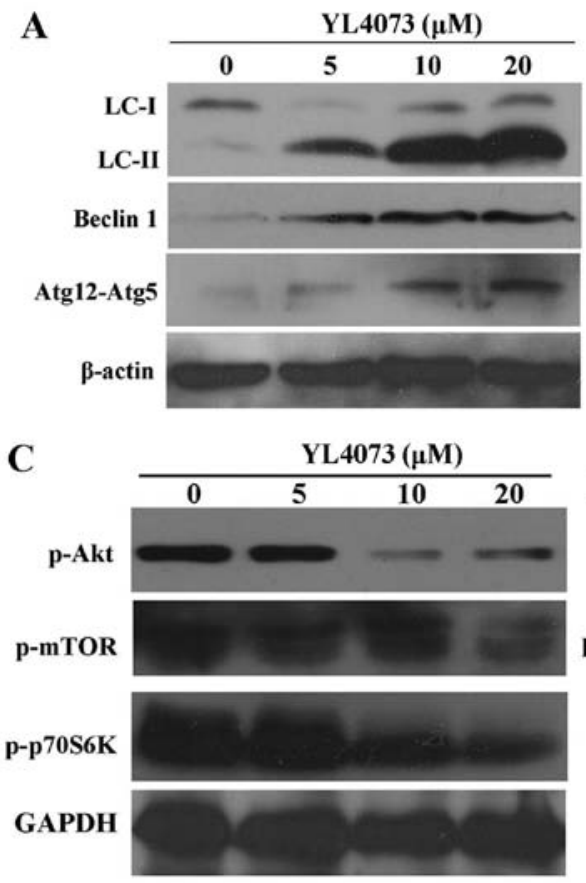

B

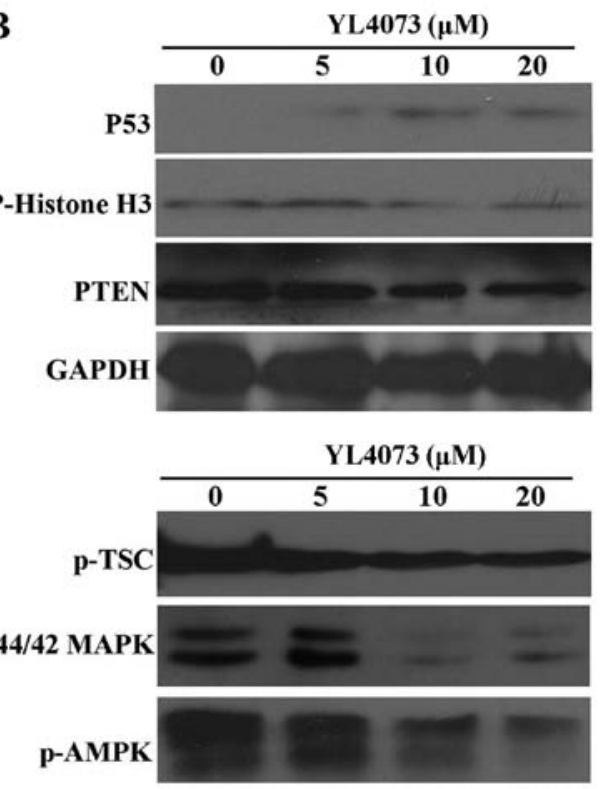

GAPDH

Figure 4. The effects of YL4073 on autophagy-associated signalling pathway were analyzed by western blotting. (A) The effect of YL4073 on autophagyrelated proteins LC3, Beclin 1, and Atg12-Atg5 complex was analyzed by western blotting. (B) The effect of YL4073 on apoptosis relative proteins P53, p-histone $\mathrm{H} 3$ was analyzed by western blotting. (C) The effect of YL4073 on the phosphorylation of proteins in Akt/mTOR/p70S6K signalling pathway. (D) The effect of YL4073 on the phosphorylation of proteins in TSC/MAPK/AMPK signalling pathway. Data are representative of three parallel experiments.

YL4073 induces autophagosome formation in LL/2 cells. Western blotting was performed to monitor the alteration of YL4073-mediated autophagy (34). As shown in Fig. 4A, LC3-II protein was detectable in LL/2 cells following YL4073 treatment for $48 \mathrm{~h}$. Activation of a series of autophagy proteins was a critical step for autophagosome formation. To determine whether the key autophagy-related proteins were synergisti- cally activated in response to YL4073-induced autophagy, the expression of Beclin 1 and the key regulator of autophagy, Atg12/Atg 5 conjugate, were assessed in LL/2 cells. As shown in Fig. 4A, YL4073 induced a marked accumulation of Beclin 1 and intracellular Atg12-Atg5 complex after $48 \mathrm{~h}$ compared with vehicle; moreover, p-histone $\mathrm{H} 3$ was significantly inhibited by YL4073, while the expression of P53 was 
A

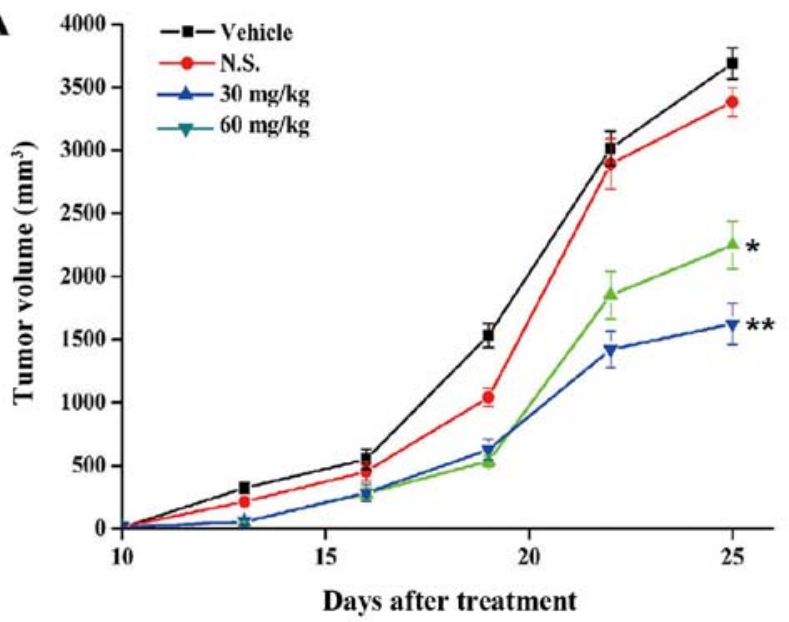

B
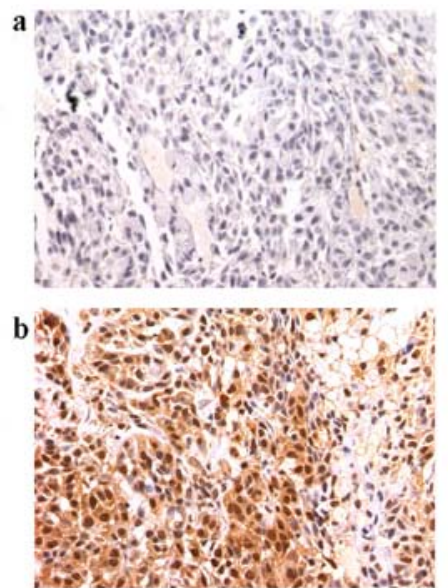

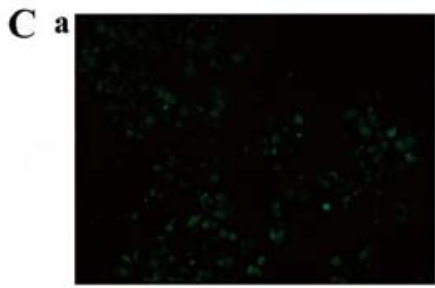

c

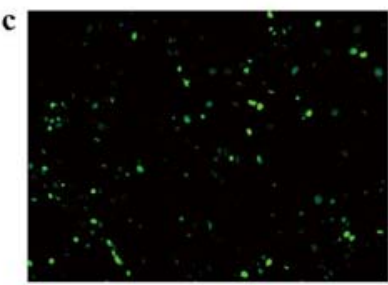

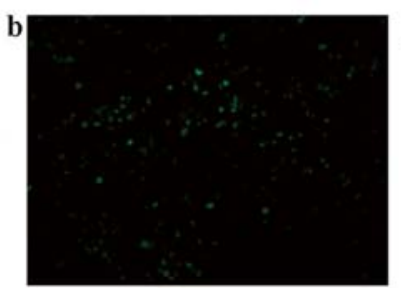

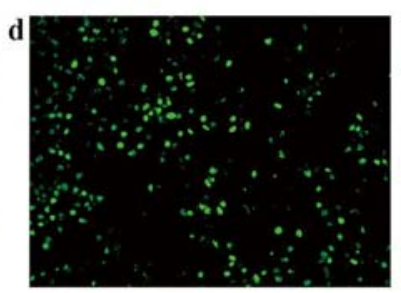

D

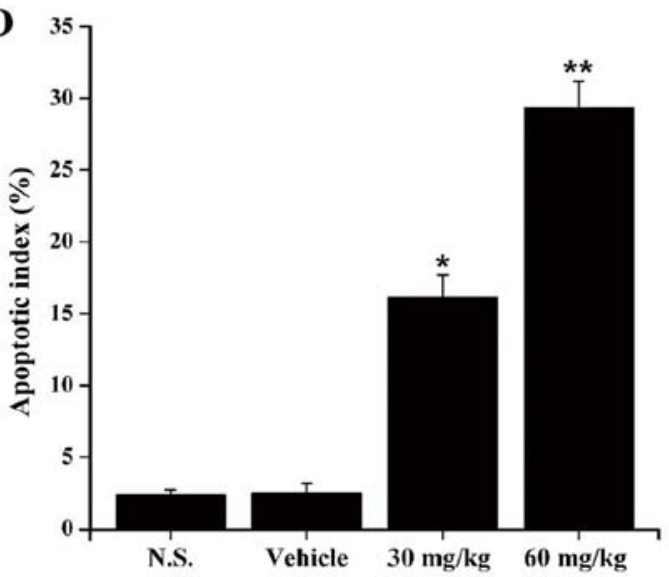

Figure 5. Treatment with YL4073 significantly attenuates the growth of mouse-bearing LL/2 cell tumor models by promoting apoptosis. (A) Tumor model: LL/2 cells were injected subcutaneously in female C57BL/6 mice. After 10 days of tumor growth, the mice received intraperitoneal administration of YL4073. Tumor volumes (mean $\pm \mathrm{SEM} ; \mathrm{n}=10 ;{ }^{*} \mathrm{P}<0.05 ;{ }^{* *} \mathrm{P}<0.01$ ). (B) Immunohistochemical analysis of xenograft tumor sections after $\mathrm{YL} 4073$ administration to mice. Tumor sections were stained with LC3-II antibody and observed under an inverted microscope; representative images of xenograft tumor sections (a) vehicle, (b) $60 \mathrm{mg} / \mathrm{kg}(\mathrm{x} 200 ; \mathrm{n}=10)$. (x200; $\mathrm{n}=10)$. (C) TUNEL analysis of xenograft tumor sections. Macroscopic view of tumor sections with TUNEL; representative fields of xenograft tumor sections (a) N.S., (b) Vehicle, (c) $30 \mathrm{mg} / \mathrm{kg}$, (d) $60 \mathrm{mg} / \mathrm{kg}$ (x200; n=10). (D) The percentage of apoptotic area (mean \pm SEM) of tumor sections as quantified by ImageJ software. Tumor apoptosis $(\%)=($ apoptotic cells $) /\left(\right.$ total cells) $\mathrm{x} 100 \%\left({ }^{*} \mathrm{P}<0.05 ;{ }^{* *} \mathrm{P}<0.01\right)$.

slightly activated (Fig. 4B). Collectively, these observations suggest that YL4073 induces autophagy and apoptosis in LL/2 cells.

YL4073 inhibits Akt/mTOR/p70S6K and activates TSC/MAPK/ AMPK pathway in LL/2 cells. Western blot analysis was also used to evaluate the effect of YL4073 on the Akt/mTOR/ p70S6K pathway, because it is the main pathway that downregulates autophagy (35). Treatment with YL4073 decreased phosphorylated Akt (p-Akt) effectively for $48 \mathrm{~h}$ in LL/2 cells; p-mTOR activity was also affected (Fig. 4C). These results suggest that the upstream pathway of Akt was influenced by YL4073 treatment. Furthermore, YL4073 decreased p-p70S6K and p-TSC gradually in LL/2 cells after $48 \mathrm{~h}$.

Because the AMPK/MAPK pathway upregulated autophagy in starved cancer cells (36), we examined the effects of YL4073 treatment on this pathway. As shown in Fig. 4D, YL4073 decreased p-AMPK and p-p44/42MAPK for $48 \mathrm{~h}$ in LL/2 cells. Collectively, these results indicated that YL4073 could inhibit both the Akt/ mTOR/p70S6K pathway and the AMPK/MAPK pathway, and both changes potentially mediated YL4073-induced autophagy.

Pharmacokinetic and pharmacodynamic profile of YL4073. YL4073 was used as an anticancer therapeutic agent model in a pharmacokinetics study. We determined certain pharmacokinetic parameters of YL4073 in blood serum of SD rats by HPLC. A concentration versus time curve was measured in serum at each time-point. Our results shown that the plasma concentration of YL4073 at $5 \mathrm{~min}$ was $5.53 \mu \mathrm{g} / \mathrm{ml}$ after treatment with a $30 \mathrm{mg} / \mathrm{kg}$ dose. Pharmacokinetic modelling suggested that the plasma concentration-time profile can be described using a two-compartment model with an estimated $\mathrm{t}_{1 / 2}$ of $4.4 \mathrm{~h}$ and urinary clearance representing $5.1 \%$ of systemic clearance, with substantial variation in the plasma concentration. The AUC was $11.9 \mathrm{mg} / \mathrm{l}$ per hour. We used AUC to estimate the efficacy of YL4073; it was also used to predict toxicity. The results indicated that YL4073 had high efficacy and low toxicity.

We evaluated whether YL4073 inhibited the growth of LL/2 subcutaneous tumors by inducing apoptosis and 
autophagy in vivo. C57BL/6 mice were inoculated subcutaneously with LL/2 cells, and after ten days, tumor volume reached about 50 to $70 \mathrm{~mm}^{3}$. Intraperitoneal injections of $60 \mathrm{mg} / \mathrm{kg} /$ day YL4073, $30 \mathrm{mg} / \mathrm{kg} /$ day YL4073, vehicle, and N.S. were administered to different groups. Tumor growth was observed for 14 days after the initiation of treatment. On day 14 , there was $29.1 \%$ and $52.6 \%$ tumor growth inhibition in $60 \mathrm{mg} / \mathrm{kg} / \mathrm{day}$ and $30 \mathrm{mg} / \mathrm{kg} /$ day YL4073 groups compared with vehicle $(\mathrm{P}<0.05$; Fig. $5 \mathrm{~A})$. In addition, immunohistochemical analysis and TUNEL assay was used to examine whether YL4073 induced autophagy and apoptosis in vivo. As shown in Fig. 5B-D, the expression levels of LC3-II increased, and the number of apoptotic cells in YL4073treated groups was more than that in the vehicle group, after treatment with YL4073 in vivo. The results were consistent with the results of WB in vitro. These results suggest that YL4073 inhibited LL/2 tumor growth in vivo by inducing autophagy and apoptosis.

\section{Discussion}

Although notable progress has been made in the management of advanced lung cancer, many challenges remain (37). Chemotherapy has been the primary treatment for patients with advanced lung cancers (38). However, recent studies suggest no significant improvement in survival rate in these patients (39). There is an urgent, unanswered medical necessity for the development of rational and effective therapies to treat advanced malignant lung cancers (40). In this study, we demonstrated that small molecular compound YL4073 induced autophagy and apoptosis in murine Lewis lung cancer LL/2 cells both in vitro and in vivo.

YL4073 has significant anti-proliferation activities against a panel of murine cancer cell lines, including 4T1 cells and LL/2 cells; LL/2 cells were most sensitive to YL4073 treatment. Furthermore, we focused on the effect of YL4073 on LL/2 cells to study the relationship between the autophagy and apoptosis pathways involved. The anti-proliferation effect of YL4073 has yet to be studied in a wider variety of cancer cells. Susceptibility to apoptosis of tumor cells was an important determinant of effective therapy.

Based on our results, we consider that YL4073 inhibits the growth of tumors by inducing autophagy. Our results indicated YL4073 induced LL/2 cell autophagy in a concentration-dependent manner. We demonstrated that YL4073 promoted an autophagic process in LL/2 cells. Our data show that YL4073 treatment resulted in the appearance of a series of autophagic markers, including development of double-membrane autophagic vacuoles and AVO augmented conversion of LC3-I to LC3-II (41). Furthermore, YL4073 treatment caused the activation of a group of autophagy-related proteins, including Beclin 1 and Atg12-Atg5 complex, both of which are essential for autophagosome formation (42).

Inhibition of the $\mathrm{Akt} / \mathrm{mTOR} / \mathrm{p} 70 \mathrm{~S} 6 \mathrm{~K}$ and the TSC/MAPK/AMPK signalling pathways may contribute to the autophagy of tumors (43). The Akt-mTOR signalling pathway is an important downregulator of autophagy (42). The study also employed EdU-DNA incorporation assays to show that YL4073 could inhibit the proliferation of tumor cells; p-Akt and p-mTOR were also inhibited in the presence of
YL4073, suggesting that inhibition of the Akt/mTOR/p70S6K signalling pathway contributed to YL4073-induced autophagy in LL/2 cells. In addition, the phosphorylation of TSC, MAPK and AMPK were inhibited by YL4073 treatment. These findings suggest that inhibition of these two signalling pathways is a potential therapeutic approach for the treatment of cancer. YL4073 effectively inhibited tumor growth in an established LL/2 lung cancer model, and induced autophagy in vivo. In addition, there were no obviously pathological changes in major organs, including the heart, liver, spleen, lung, kidney and brain, according to H\&E stain (data not shown).

We also attempted to identify the primary target of YL4073, since it is critical to further study. We have carried out some limited research to this end; a bioinformatics-based 'reverse docking' method with computer-aided drug designed has been applied, which predicted that the possible primary targets might be CXCR3, glutathione S-transferase, chymotrypsin, and penicillopepsin. Furthermore, the results of the kinase inhibitory activity experiment showed that YL4073 did not block kinase activity of MEK, or Raf (12). Therefore, the primary molecular target of YL4073 is not yet identified. Our aim for further study is to use new approaches to identify the primary target, such as quantitative chemical proteomics, which has been used as an effective method to detect the primary molecular target of some agents (44).

In conclusion, we demonstrated that YL4073 has anticancer activity and that it induced autophagy and apoptosis both in vitro and in vivo. The anticancer mechanisms involved LC3, Beclin 1, and Atg5/Atg12 complexes, inhibition of the Akt/mTOR/p70S6K pathway, and activation of the AMPK/MAPK pathway in LL/2 cells. These results indicate that YL4073 warrants further in-depth study and may be a promising agent for future lung cancer therapy.

\section{Acknowledgements}

This study was supported by the National Natural Sciences Foundation of China (no. 81272459 and 81402947), Natural Sciences Foundation of Anhui Province (1508085QH162), China Postdoctoral Science Foundation Funded Project (2015M581974), and Grants for Scientific Research of BSKY from Anhui Medical University (XJ201315).

\section{References}

1. Parkin DM, Bray F, Ferlay J and Pisani P: Estimating the world cancer burden: Globocan 2000. Int J Cancer 94: 153-156, 2001.

2. Tyczynski JE, Bray F and Parkin DM: Lung cancer in Europe in 2000: Epidemiology, prevention, and early detection. Lancet Oncol 4: 45-55, 2003.

3. Siegel R, Naishadham D and Jemal A: Cancer statistics, 2013. CA Cancer J Clin 63: 11-30, 2013.

4. Singh RP, Deep G, Chittezhath M, Kaur M, Dwyer-Nield LD, Malkinson AM and Agarwal R: Effect of silibinin on the growth and progression of primary lung tumors in mice. J Natl Cancer Inst 98: 846-855, 2006.

5. Proctor RN: Tobacco and the global lung cancer epidemic. Nat Rev Cancer 1: 82-86, 2001.

6. Li ZG, Zhao YL, Wu X, Ye HY, Peng A, Cao ZX, Mao YQ, Zheng YZ, Jiang PD, Zhao X, et al: Barbigerone, a natural isoflavone, induces apoptosis in murine lung-cancer cells via the mitochondrial apoptotic pathway. Cell Physiol Biochem 24: 95-104, 2009.

7. Gill RR, Jaklitsch MT and Jacobson FL: Controversies in lung cancer screening. J Am Coll Radiol 10: 931-936, 2013. 
8. De la Cruz CS, Tanoue LT and Matthay RA: Lung cancer: Epidemiology, etiology, and prevention. Clin Chest Med 32: 605-644, 2011.

9. Ettinger DS, Akerley W, Borghaei H, Chang AC, Cheney RT, Chirieac LR, D'Amico TA, Demmy TL, Govindan R, Grannis FW Jr, et al; National comprehensive cancer network: Non-small cell lung cancer, version 2.2013. J Natl Compr Canc Netw 11: 645-653, quiz 653, 2013.

10. Llovet JM, Ricci S, Mazzaferro V, Hilgard P, Gane E, Blanc JF, de Oliveira AC, Santoro A, Raoul JL, Forner A, et al; SHARP Investigators Study Group: Sorafenib in advanced hepatocellular carcinoma. N Engl J Med 359: 378-390, 2008.

11. Motzer RJ, Hutson TE, Tomczak P, Michaelson MD, Bukowski RM, Rixe O, Oudard S, Negrier S, Szczylik C, Kim ST, et al: Sunitinib versus interferon alfa in metastatic renal-cell carcinoma. N Engl J Med 356: 115-124, 2007.

12. Xu YZ, Zheng RL, Zhou Y, Peng F, Lin HJ, Bu Q, Mao YQ, Yu LT, Yang L, Yang SY, et al: Small molecular anticancer agent SKLB703 induces apoptosis in human hepatocellular carcinoma cells via the mitochondrial apoptotic pathway in vitro and inhibits tumor growth in vivo. Cancer Lett 313: 44-53, 2011

13. Yue Z, Jin S, Yang C, Levine AJ and Heintz N: Beclin 1, an autophagy gene essential for early embryonic development, is a haploinsufficient tumor suppressor. Proc Natl Acad Sci USA 100: 15077-15082, 2003

14. Eisenberg-Lerner A, Bialik S, Simon HU and Kimchi A: Life and death partners: Apoptosis, autophagy and the cross-talk between them. Cell Death Differ 16: 966-975, 2009.

15. Kuma A, Hatano M, Matsui M, Yamamoto A, Nakaya H, Yoshimori T, Ohsumi Y, Tokuhisa T and Mizushima N: The role of autophagy during the early neonatal starvation period. Nature 432: 1032-1036, 2004.

16. 16. Lum JJ, Bauer DE, Kong M, Harris MH, Li C, Lindsten T and Thompson CB: Growth factor regulation of autophagy and cell survival in the absence of apoptosis. Cell 120: 237-248, 2005.

17. Klionsky DJ and Emr SD: Autophagy as a regulated pathway of cellular degradation. Science 290: 1717-1721, 2000.

18. Ohsumi Y: Molecular dissection of autophagy: Two ubiquitin-like systems. Nat Rev Mol Cell Biol 2: 211-216, 2001.

19. Shintani T and Klionsky DJ: Autophagy in health and disease: A double-edged sword. Science 306: 990-995, 2004.

20. White E and DiPaola RS: The double-edged sword of autophagy modulation in cancer. Clin Cancer Res 15: 5308-5316, 2009.

21. Gozuacik D and Kimchi A: Autophagy as a cell death and tumor suppressor mechanism. Oncogene 23: 2891-2906, 2004.

22. Amaravadi RK and Thompson CB: The roles of therapy-induced autophagy and necrosis in cancer treatment. Clin Cancer Res 13 7271-7279, 2007.

23. Kondo Y, Kanzawa T, Sawaya R and Kondo S: The role of autophagy in cancer development and response to therapy. Nat Rev Cancer 5: 726-734, 2005.

24. Espert L, Denizot M, Grimaldi M, Robert-Hebmann V, Gay B, Varbanov M, Codogno P and Biard-Piechaczyk M: Autophagy is involved in T cell death after binding of HIV-1 envelope proteins to CXCR4. J Clin Invest 116: 2161-2172, 2006.

25. Bommareddy A, Hahm ER, Xiao D, Powolny AA, Fisher AL, Jiang Y and Singh SV: Atg5 regulates phenethyl isothiocyanate-induced autophagic and apoptotic cell death in human prostate cancer cells. Cancer Res 69: 3704-3712, 2009.

26. Kanzawa T, Kondo Y, Ito H, Kondo S and Germano I: Induction of autophagic cell death in malignant glioma cells by arsenic trioxide. Cancer Res 63: 2103-2108, 2003.

27. Abe J, Kusuhara M, Ulevitch RJ, Berk BC and Lee JD: Big mitogen-activated protein kinase 1 (BMK1) is a redox-sensitive kinase. J Biol Chem 271: 16586-16590, 1996.

28. Buck E, Eyzaguirre A, Brown E, Petti F, McCormack S, Haley JD, Iwata KK, Gibson NW and Griffin G: Rapamycin synergizes with the epidermal growth factor receptor inhibitor erlotinib in non-small-cell lung, pancreatic, colon, and breast tumors. Mol Cancer Ther 5: 2676-2684, 2006.
29. Bursch W, Ellinger A, Gerner C, Fröhwein U and Schulte-Hermann R: Programmed cell death (PCD). Apoptosis, autophagic PCD, or others? Ann NY Acad Sci 926: 1-12, 2000.

30. Bursch W, Hochegger K, Torok L, Marian B, Ellinger A and Hermann RS: Autophagic and apoptotic types of programmed cell death exhibit different fates of cytoskeletal filaments. J Cell Sci 113: 1189-1198, 2000

31. Traganos F and Darzynkiewicz Z: Lysosomal proton pump activity: Supravital cell staining with acridine orange differentiates leukocyte subpopulations. Methods Cell Biol 41: 185-194, 1994.

32. Paglin S, Hollister T, Delohery T, Hackett N, McMahill M, Sphicas E, Domingo D and Yahalom J: A novel response of cancer cells to radiation involves autophagy and formation of acidic vesicles. Cancer Res 61: 439-444, 2001.

33. Kanzawa T, Germano IM,Komata T, Ito H, Kondo Y and Kondo S: Role of autophagy in temozolomide-induced cytotoxicity for malignant glioma cells. Cell Death Differ 11: 448-457, 2004.

34. Kim J and Klionsky DJ: Autophagy, cytoplasm-to-vacuole targeting pathway, and pexophagy in yeast and mammalian cells. Annu Rev Biochem 69: 303-342, 2000.

35. Shigemitsu K, Tsujishita Y, Hara K, Nanahoshi M, Avruch J and Yonezawa K: Regulation of translational effectors by amino acid and mammalian target of rapamycin signaling pathways. Possible involvement of autophagy in cultured hepatoma cells. J Biol Chem 274: 1058-1065, 1999.

36. Pattingre $\mathrm{S}$, Bauvy $\mathrm{C}$ and Codogno $\mathrm{P}$ : Amino acids interfere with the ERK1/2-dependent control of macroautophagy by controlling the activation of Raf-1 in human colon cancer HT-29 cells. J Biol Chem 278: 16667-16674, 2003.

37. Sandler A, Gray R, Perry MC, Brahmer J, Schiller JH, Dowlati A, Lilenbaum R and Johnson DH: Paclitaxel-carboplatin alone or with bevacizumab for non-small-cell lung cancer. N Engl J Med 355: 2542-2550, 2006

38. Scagliotti GV, Parikh P, von Pawel J, Biesma B, Vansteenkiste J, Manegold C, Serwatowski P, Gatzemeier U, Digumarti R, Zukin M, et al: Phase III study comparing cisplatin plus gemcitabine with cisplatin plus pemetrexed in chemotherapy-naive patients with advanced-stage non-small-cell lung cancer. J Clin Oncol 26: 3543-3551, 2008

39. Luistro L, He W, Smith M, Packman K, Vilenchik M, Carvajal D, Roberts J, Cai J, Berkofsky-Fessler W, Hilton H, et al: Preclinical profile of a potent $\gamma$-secretase inhibitor targeting notch signaling with in vivo efficacy and pharmacodynamic properties. Cancer Res 69: 7672-7680, 2009.

40. Karna P, Zughaier S, Pannu V, Simmons R, Narayan S and Aneja R: Induction of reactive oxygen species-mediated autophagy by a novel microtubule-modulating agent. J Biol Chem 285: 18737-18748, 2010.

41. Jiang H, White EJ, Ríos-Vicil CI, Xu J, Gomez-Manzano C and Fueyo J: Human adenovirus type 5 induces cell lysis through autophagy and autophagy-triggered caspase activity. J Virol 85: 4720-4729, 2011.

42. Shinojima N, Yokoyama T, Kondo Y and Kondo S: Roles of the Akt/mTOR/p70S6K and ERK1/2 signaling pathways in curcumin-induced autophagy. Autophagy 3: 635-637, 2007.

43. Codogno P and Meijer AJ: Autophagy and signaling: Their role in cell survival and cell death. Cell Death Differ 12 (Suppl 2): $1509-1518,2005$

44. Li J, Rix U, Fang B, Bai Y, Edwards A, Colinge J, Bennett KL, Gao J, Song L, Eschrich S, et al: A chemical and phosphoproteomic characterization of dasatinib action in lung cancer. Nat Chem Biol 6: 291-299, 2010. 